University of Warwick institutional repository

This paper is made available online in accordance with

publisher policies. Please scroll down to view the document itself. Please refer to the repository record for this item and our policy information available from the repository home page for further information.

To see the final version of this paper please visit the publisher's website. Access to the published version may require a subscription.

Authors:

Title:

Year of

publication:

Link to published version:

Publisher

statement:
Robert Zellem, Nicholas Hollon, RonaldLOUIS BALlOUZ, EDWARD M. SION, AND PATRICK GODON, BORIS T. GÄNSICKE and KNOX LONG Hubble Space Telescope STIS Spectroscopy of the Peculiar NovaLike Variables BK Lyn, V751 Cygni, and V380 Op 2009

http://dx.doi.org/10.1086/605547

None 


\title{
Hubble Space Telescope STIS Spectroscopy of the Peculiar Nova-Like Variables BK Lyn, V751 Cygni, and V380 Oph
}

\author{
Robert Zellem, Nicholas Hollon, Ronald-Louis Ballouz, Edward M. Sion, and Patrick Godon ${ }^{1}$ \\ Dept of Astronomy \& Astrophysics, Villanova University, Villanova, PA 19085; robert.zellem@villanova.edu, \\ nicholas.hollon@villanova.edu, \\ ronald-louis.ballouz@villanova.edu, edward.sion@villanova.edu, patrick.godon@villanova.edu \\ BORIS T. GÄNSICKE \\ Department of Physics, University of Warwick, Coventry CV4 9BU, UK; Boris.Gaensicke@warwick.ac.uk \\ AND \\ KNOX LONG \\ Space Telescope Science Institute, Baltimore, MD 21208; long@stsci.edu \\ Received 2008 November 8; accepted 2009 June 24; published 2009 August 14
}

\begin{abstract}
We obtained Hubble STIS spectra of three nova-like variables: V751 Cygni, V380 Oph, and - the only confirmed nova-like variable known to be below the period gap-BK Lyn. In all three systems, the spectra were taken during high optical brightness state, and a luminous accretion disk dominates their farultraviolet (FUV) light. We assessed a lower limit of the distances by applying the infrared photometric method of Knigge. Within the limitations imposed by the poorly known system parameters (such as the inclination, white dwarf mass, and the applicability of steady state accretion disks) we obtained satisfactory fits to BK Lyn using optically thick accretion disk models with an accretion rate of $\dot{M}=1 \times 10^{-9} M_{\odot} \mathrm{yr}^{-1}$ for a white dwarf mass of $M_{\text {wd }}=1.2 M_{\odot}$ and $\dot{M}=1 \times 10^{-8} M_{\odot} \mathrm{yr}^{-1}$ for $M_{\text {wd }}=0.4 M_{\odot}$. However, for the VY Scl-type nova-like variable V751 Cygni and for the SW Sex star V380 Oph, we are unable to obtain satisfactory synthetic spectral fits to the high state FUV spectra using optically thick steady state accretion disk models. The lack of FUV spectra information down to the Lyman limit hinders the extraction of information about the accreting white dwarf during the high states of these nova-like systems.
\end{abstract}

Online material: color figures

\section{INTRODUCTION}

Cataclysmic variables (CVs) are short-period binaries in which a late-type, Roche lobe-filling main-sequence dwarf transfers gas through an accretion disk onto a rotating, accretion-heated white dwarf (WD). The nova-like variables are a subclass of CVs in which the mass-transfer rate is high and the light of the system is dominated by a very bright accretion disk. The spectra of nova-like variables resemble those of classical novae $(\mathrm{CN})$ that have settled back to quiescence. However, nova-like variables have never had a recorded CN outburst. Hence their evolutionary status is unknown. They could be close to having their next $\mathrm{CN}$ explosion, or they may have had an unrecorded explosion, possibly hundreds or thousands of years ago. Adding to the mystery of nova-like variables is that some of them (known as the VY Sculptoris

\footnotetext{
${ }^{1}$ Visiting at the Space Telescope Science Institute, Baltimore, MD 21208; godon@stsci.edu.
}

stars after their prototype) show the curious behavior of being in a high optical brightness state most of the time, but then, plummeting into a deep low-brightness state with little or no ongoing accretion. Then, just as unpredictably, they return to the high-brightness state. These drops are possibly related to cessation of mass transfer from the K-M dwarf secondary star either by starspots positioned under L1 (Livio \& Pringle 1994) or irradiation feedback in which an inflated outer disk can modulate the mass transfer from the secondary by blocking irradiation of the hot inner accretion disk region (Wu et al. 1995).

As part of a Hubble Space Telescope Snapshot project extending over three Hubble observing cycles (B. Gänsicke, PI), several nova-like variables were observed among dozens of nonmagnetic and magnetic cataclysmic variables. In this article we report on an analysis of the HST Space Telescope Imaging Spectrograph (STIS) spectra of three of these objects, BK Lyn, V751 Cygni, and V380 Oph. 


\subsection{BK Lyn}

BK Lyn (PG0917+342) is an extraordinary nova-like variable with an orbital period placing it below the CV period gap. As such, it is the only bona fide nova-like variable below the CV period gap (Dobrzycka \& Howell 1992). It was classified a short period dwarf nova by Dhillon et al. (2000) but long-term light curves from ROBOSCOPE (Ringwald et al. 1996), comprising 98 exposures over $3 \mathrm{yr}$, and the Harvard Plate Collection reveal no evidence for dwarf nova outbursts or even low optical brightness states. The system normally remains between $\mathrm{V}=14.6$ and 14.7. Ringwald et al. (1996) confirmed that its orbital period places it below the period gap. They carried out an $\mathrm{H} \alpha$ radial velocity study of BK Lyn which revised its orbital period and determined the radial velocity semiamplitude $\mathrm{K}$ of 70 to $130 \mathrm{~km} \mathrm{~s}^{-1}$ Ringwald et al. (1996). Skillman \& Patterson (1993) detected permanent superhumps and found that the optical spectrum fits a power law $f_{\lambda}=k_{\lambda}^{-\alpha}$ of index $\alpha=2.66 \pm 0.10$, characteristic of a steady state accretion disk. Dhillon et al. (2000) carried out infrared spectrophotometry of BK Lyn that gave an upper limit to the contribution of an M5V secondary to the K-band light of $50 \% \pm 5 \%$. Dobrzycka \& Howell (1992) estimated the binary's inclination to be $32 \pm 12$ degrees, WD mass of $0.3_{-0.12}^{+0.35}$. However, the WD mass is not well-constrained. Sproats et al. (1996) give a distance estimate of 114 pc using K magnitudes and Bailey's method, while Dhillon et al. (2000) give a lower limit of 185 pc using IR spectroscopy. The distance to the system is crucial for this analysis. Puebla et al. (2007) cite a distance of $150 \mathrm{pc}$ which appears to be the average of the Sproats et al. (1996) distance and the Dhillon et al. (2000) lower limit of 185 pc. Puebla et al. (2007) used a statistical multiparameter optimization modeling technique to derive an accretion rate for BK Lyn but this value was based upon a fixed adopted distance of $150 \mathrm{pc}$. We will further discuss their result in $\S 5$ below. Dobrzycka \& Howell (1992) gave $\sim 1000 \mathrm{pc}$ but based it on assuming the average absolute visual magnitude for nova-like variables of +4.0 . We have used the method of Knigge (2006) to obtain a strict lower limit distance of $116 \mathrm{pc}$.

\subsection{V751 Cygni}

V751 Cygni is classified as a VY Scl system. A far ultraviolet IUE spectrum of V751 Cygni was taken on 1985 April 25 when the system was at or near optical maximum. The spectrum, SWP25574, was obtained at low dispersion through the large aperture. The visual magnitude with the fine error sensor (FES) was $\mathrm{V}(\mathrm{FES})=14.2$ while a longer wavelength UV spectrum, LWP05819, yielded an interstellar reddening value $\mathrm{E}(B-V)=0.25 \pm 0.05$ from the $2200 \AA$ A feature (Greiner et al. 1999). The large amount of reddening introduces additional uncertainty. Based upon the lack of emission lines and upon a study by Greiner (1998) of equivalent width versus orbital inclinations for VY Scl stars, Greiner et al. (1999) concluded that a low inclination $\left(<50^{\circ}\right)$ is indicated. They estimate from the Greiner (1998) study that $i=30^{\circ} \pm 20^{\circ}$. Greiner et al. (1999) do mention a possible He II (1640) absorption feature. In fact, this feature, if real, is the strongest absorption line in the IUE spectrum. However, while Greiner et al. (1999) measured equivalent widths for the $\mathrm{SiV}, \mathrm{C} \mathrm{IV}$, and $\mathrm{N} \mathrm{V}$, which are only marginally present in the IUE spectrum, they do not give further mention to the strongest absorption feature near $1640 \AA$.

The donor star was classified early to mid-F star, which was implied by the presence of the G-band and $\mathrm{Na} \mathrm{D}$ lines with absence of Mg b 5169A (Downes et al. 1995). However, subsequent work demonstrated that the feature thought to be the G-band was actually part of the $\mathrm{H} \gamma$ wing while the $\mathrm{Na}$ D lines proved to be interstellar. There are also reports of transient optical P Cygni absorption which appears correlated with binary phase (Patterson et al. 2001). The mass of the WD is unknown. The radial velocity studies done to date using disk emission lines, which may reflect the dynamical motion of the WD, have been inconclusive (Walker \& Bell 1980; Echevarría et al. 2002; Downes et al. 1995).

Greiner et al. (1999) carried out a photometric and spectroscopic analysis of the system and reported negative superhumps and the unexpected detection of soft X-rays instead of the predicted hard X-rays, during the low optical brightness state of V751 Cygni when the boundary layer of the WD is optically thin. They suggest that V751 Cyg is a supersoft X-ray binary, with a WD undergoing mass accretion at a high enough rate to cause quasi-steady hydrogen burning on the surface. This would imply that the WD is highly luminous $\left(\mathrm{L}_{\mathrm{bol}} \sim 10^{36}-\right.$ $10^{38} \mathrm{ergs} \mathrm{s}^{-1}$ ). Patterson et al. (2001) confirm the soft X-ray detection during quiescence but contend that V751 Cyg is not a supersoft binary, but has a low luminosity soft X-ray component $\left(\sim 10^{33} \mathrm{ergs} \mathrm{s}^{-1} \AA\right)$. Clearly, the mass of the WD in this systems is critical. V751 Cyg could also be an SW Sextantis star as well. SW Sextantis systems, another nova-like subclass, are characterized by a multitude of observational characteristics: orbital periods between 3 and $4 \mathrm{hr}$, one-third of the systems noneclipsing and two-thirds showing deep eclipses of the WD by the secondary, thus requiring high inclination angles, single-peaked emission lines despite the high inclination, and high excitation spectral features including He II (4686) emission and strong Balmer emission on a blue continuum, high-velocity emission $\mathrm{s}$-waves with maximum blueshift near phase $\sim 0.5$, delay of emission line radial velocities relative to the motion of the WD, and cental absorption dips in the emission lines around phase $\sim 0.4-0.7$ (Rodriguez-Gil et al. (2007; Hoard et al. 2003). The WDs in many, if not all, of these systems are suspected of being magnetic (Rodriguez-Gil et al. 2007). V751 Cyg has the right orbital period as well as negative superhumps in the system to possibly be an SW Sex star. It is important therefore to explore the physical properties of the disk and, if possible, underlying, accreting WD in V751 Cygni. 


\subsection{V380 Oph}

The third system, V380 Oph, is classified as a definite SW Sex member. The WDs in many, if not all, of these systems are suspected of being magnetic (Rodriguez-Gil et al. 2007). Since these objects are found near the upper boundary of the period gap (Warner (1995) and references therein), their study is of critical importance to understanding $\mathrm{CV}$ evolution as they enter the period gap.

Shafter (1983) determined a spectroscopic period and radial velocity $\mathrm{K}$ value of $100 \mathrm{~km} \mathrm{~s}^{-1}$ for V380 Oph from observations with very moderate spectral resolution. Rodriguez-Gil et al. (2007) improved its orbital period and measured $\mathrm{H} \alpha$ emission radial velocity variations with an amplitude of $400 \mathrm{~km} \mathrm{~s}^{-1}$. Given the difference in radial velocity amplitudes derived by these two groups, one suspects there is considerable uncertainty in the correct value of inclination implied by these studies. Photometric observations have revealed orbital variability with possible negative superhumps.

\subsection{Reddening Values and Distances}

The reddening of the systems was taken from available estimates in the literature. The three principal sources of reddening for cataclysmic variables are the compilations of Verbunt (1987); La Dous (1991); Bruch \& Engel (1994). For V751 Cygni, Greiner et al. (1999) gave $\mathrm{E}(B-V)=0.25 \pm 0.05$ which is the value we adopted for V751 Cygni. For V380 Oph, we treated the reddening as a free parameter in the model fitting. The FUV spectra of V751 Cygni objects were dereddened using the IUERDAF IDL routine UNRED. The spectrum of BK Lyn was not dereddened as the galactic reddening in the direction of BK Lyn is very small $(\mathrm{E}(B-V) \sim 0.01$. The observed properties of all three systems are summarized in Table 1.

We have computed strict lower limit distances to BK Lyn, V751 Cygni, and V380 Oph using a method by Knigge (2006) which uses 2MASS JHK photometry. For each system, we obtained the $J, H, K$ apparent magnitudes from 2MASS. For a given orbital period, Knigge (2006) provides absolute $J, H$, and $K$ magnitudes based upon his semiempirical donor sequence for $\mathrm{CV}$ s. If it is assumed that the donor provides $100 \%$ of the light in $J, H$, and $K$, then the distance is a strict lower limit. If the donor emits $33 \%$ of the light (the remainder being accretion light), then a very approximate upper limit is obtained. At the K-band, the latter limit is a factor of 1.75 times the lower limit distance. The lower limit distances are used as constraints in the synthetic spectral fitting procedures described in $\S 2$. For BK Lyn, this leads to a lower limit distance of 116 pc. For V380 Oph, this method yields a range of distance from $293 \mathrm{pc}$ (strict lower limit) to $513 \mathrm{pc}$, while for V751 Cygni, we obtained a range from $201 \mathrm{pc}$ (strict lower limit) to $352 \mathrm{pc}$.

\section{OBSERVATIONS AND DATA REDUCTION}

FUV spectroscopy of BK Lyn, V751 Cygni, and V380 Oph was obtained with HST/STIS during HST Cycle 11. The data were obtained using the G140L grating and the $52^{\prime \prime} \times 0.2^{\prime \prime}$ aperture, providing a spectral resolution of $R \sim 1000$ over the wavelength range $1140-1720 \AA$. Since the total time involved in each snapshot observation was short ( $<35$ minutes), the observations were made in the ACCUM mode in order to minimize the instrument overheads. This resulted in exposure times of 600 to $900 \mathrm{~s}$. Each snapshot observation resulted in a single timeaveraged spectrum of each system. All of the spectral data were processed with IRAF using CALSTIS V2.13b. During target acquisition, HST points at the nominal target coordinates and takes a $5^{\prime \prime} \times 5^{\prime \prime} \mathrm{CCD}$ image with an exposure time of a few seconds. Subsequently, a small slew is performed that centers the target in the acquisition box, and a second CCD image is taken. The acquisition images for these observations were obtained using the F28 $\times 50$ LP long-pass filter, which has a central wavelength of 7228.5 and a full-width at half maximum (FWHM) of 2721.6 ̊ (Araujo-Betancor et al. 2005).

The instrumental setup and exposure details of the HST STIS spectra of BK Lyn, V380 Oph, and V751 Cygni are provided in the observing log given in Table 2; the entries are by column: (1) Target, (2) Data ID, (3) Instrument Config/ Mode, (4) Grating, (5) Aperture, (6) Date of Observation (yyyy-mm-dd), (7) Time of Observation, and (8) Exposure Time (s).

\subsection{BK Lyn}

Until 2003, there were no FUV spectra of BK Lyn with which to check for P Cygni profiles indicating wind outflow or carry out an analysis of the FUV spectral slope or FUV line

TABLE 1

System PARAMETERS

\begin{tabular}{lcccccccc}
\hline \hline & & \multicolumn{7}{c}{$i$} \\
System \& References & $\mathrm{V}_{\max }$ & $\mathrm{V}_{\min }$ & $\mathrm{P}_{\text {orb }}$ (days) & $\left({ }^{\circ}\right)$ & $\mathrm{E}(B-V)$ & $\mathrm{M}_{\mathrm{wd}}\left(\mathrm{M}_{\odot}\right)$ & $\mathrm{MM}_{2}\left(\mathrm{M}_{\odot}\right)$ & $\begin{array}{c}d \\
(\mathrm{pc})\end{array}$ \\
\hline BK Lyn $(1,2,3)$ & 14.6 & 15.3 & 0.075 & $32 \pm 12$ & $\ldots$ & $0.3:$ & $\ldots$ & $116-291$ \\
V380 Oph $(4,5,6)$ & 14.3 & $>17$ & 0.154107 & $42 \pm 13$ & $\ldots$ & $0.58 \pm 0.19$ & $0.36 \pm 0.04$ & $293-513$ \\
V751 Cyg (7, 8) & 13.2 & 17.8 & 0.144584 & $30 \pm 20$ & $0.25 \pm 0.05$ & $\ldots$ & $\ldots$ & $201-352$ \\
\hline
\end{tabular}

REFERENCES.-(1) Dobrzycka \& Howell (1992); (2) Ringwald et al. (1996); (3) Skillman \& Patterson (1993); (4) Shafter (1983); (5) Shugarov et al. (2005); (6) Rodriguez-Gil et al. (2007); (7) Echevarría et al. (2002); (8) Greiner et al. (1999). 
TABLE 2

HubBLE OBSERVATIONS

\begin{tabular}{|c|c|c|c|c|c|c|}
\hline Target & Data ID & Config/Mode & Grating & Observation Date & Observation Time & $t_{\exp }(s)$ \\
\hline BK Lyn & O6LI34010 & STIS/FUV-MAMA/ACCUM & G140L & 2003-04-13 & $01: 12: 43$ & 600 \\
\hline V380 Oph ..... & O6L186010 & STIS/FUV-MAMA/ACCUM & G140L & 2003-07-06 & $05: 53: 14$ & 730 \\
\hline V751 Cyg ..... & O6LI1D010 & STIS/FUV-MAMA/ACCUM & G140L & 2002-12-01 & $17: 11: 16$ & 700 \\
\hline
\end{tabular}

profiles. As part of an HST snapshot program (see § 2), the first FUV spectrum of BK Lyn was secured. The spectrum is displayed in Fig. 1 where a steeply rising continuum toward shorter wavelengths is seen together with strong emission features at C III (1175), NV (1240), Si III + OI (1300), C II (1335), Si IV (1400), C IV (1550), and weak He II (1640) emission. The emission lines suggest it is probable that an accretion disk is present in the system at the time of our HST spectrum. The continuum flux level ranges from $\sim 2 \times 10^{-14} \mathrm{ergs} / \mathrm{cm}^{2} / \mathrm{s} / \AA$ at the short end to $\sim 2 \times 10^{-14} \mathrm{ergs} / \mathrm{cm}^{2} / \mathrm{s} / \AA$ at the long wavelength end. The presence of strong emission lines coupled with the low flux level and the steeply rising continuum are not characteristic of the FUV spectra of nova-like variables in their high optical brightness states unless the inclination angle is high. We note that the emission lines and continuum slope in BK Lyn's spectrum are strikingly similar in appearence to the HST and HUT spectra of the dwarf nova SS Cygni in quiescence (see Fig. 4 in Long et al. 2005) as well as the FUV spectrum of V794 Aql in its high state (Godon et al. 2007). The F $28 \times 50$ LP magnitude from the acquisition exposure was 15.3, roughly consistent with a high state but slightly fainter than the normal visual magnitude range of 14.6 to 14.7 . The difference could be due to the fact that the HST filter used in the acquisition is much redder than $\mathrm{V}$.

\subsection{V751 Cygni}

The STIS spectrum of V751 Cygni reveals moderately strong absorption features seen against a continuum rising toward shorter wavelengths. The FUV continuum even after dereddening, has a relatively flat slope. This poses a considerable challenge in finding an accretion disk spectral energy distribution which satisfies both the continuum slope and yields the observed Ly $\alpha$ line width in a single disk model. The observed width of Ly $\alpha$ should be associated with a much steeper

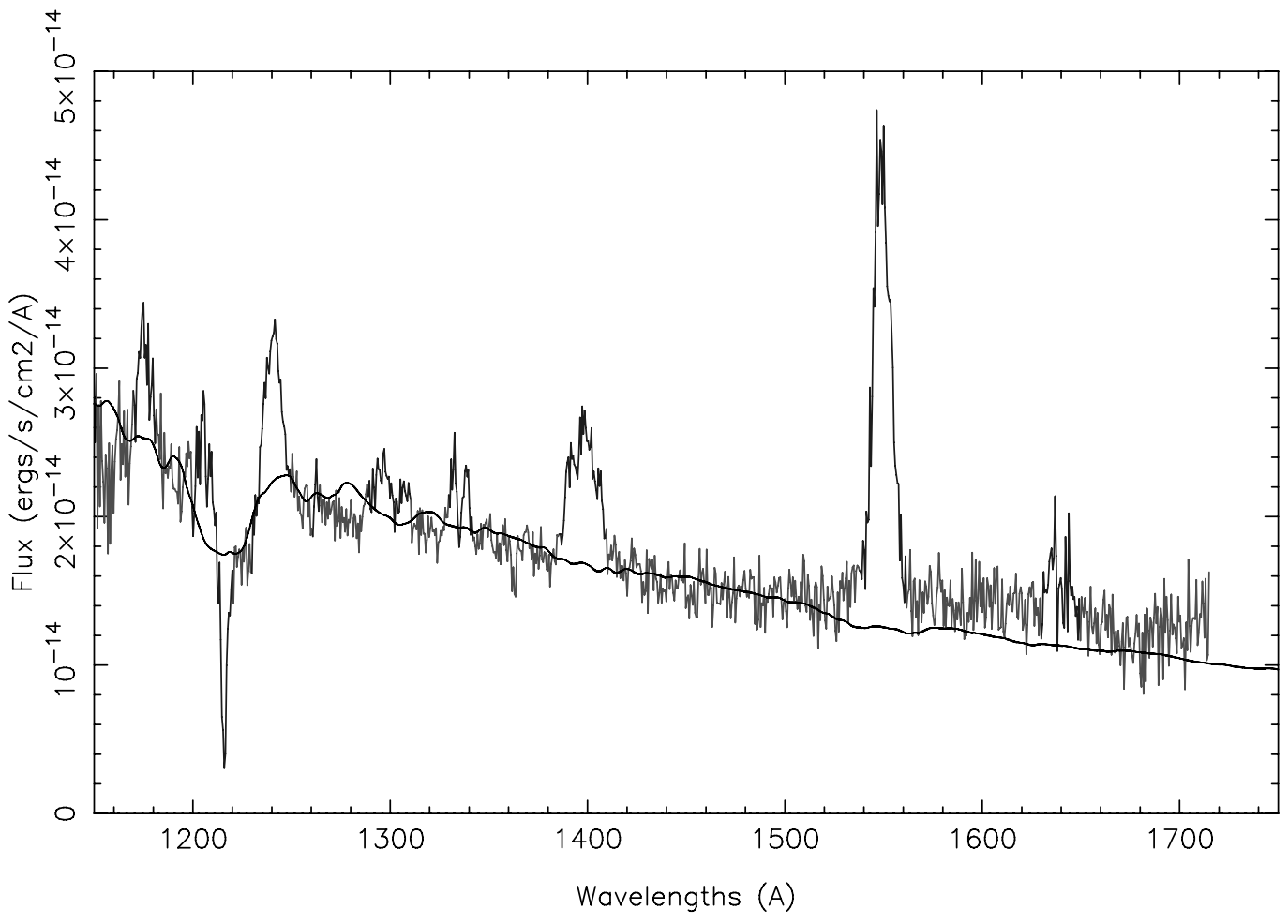

FIG. 1. - Best fitting accretion disk model fit to the Hubble STIS spectrum of BK Lyn. This fit yields $\dot{M}=1 \times 10^{-9.0}$ with $d=300$ pc, $M_{\text {wd }}=1.2 M_{\odot}$, and $i=75^{\circ}$. See the electronic edition of the PASP for a color version of this figure. 
continuum slope than is observed in the spectra. Our HST STIS spectrum confirms the identification of He II (1640) in absorption although the equivalent width of the feature is smaller than seen in the IUE spectrum (see $\S 1.1$ ). The C IV (1550) absorption line reveals a hint of P Cygni structure, probably indicating wind outflow at the time the STIS spectrum was obtained.

\subsection{V380 Oph}

The STIS spectrum of V380 Oph is dominated by strong broad-emission features superimposed on a continuum rising toward shorter wavelengths. Several weak, sharp absorption features are most likely of interstellar origin. For V380 Oph, we found the same inconsistency between the observed width of the Ly $\alpha$ profile and the observed continuuum slope. This led us to first explore the effect of interstellar reddening on the spectrum.

In Table 3, we have listed the strongest spectral features detected for each system.

\section{MULTICOMPONENT SYNTHETIC SPECTRAL MODELS}

We adopted model accretion disks from the optically thick disk model grid of Wade \& Hubeny (1998). In these accretion disk models, the innermost disk radius, $R_{\text {in }}$, is fixed at a fractional WD radius of $x=R_{\mathrm{in}} / R_{\mathrm{wd}}=1.05$. The outermost disk radius, $R_{\text {out }}$, was chosen so that $T_{\text {eff }}\left(R_{\text {out }}\right)$ is near $10,000 \mathrm{~K}$, since disk annuli beyond this point, which are cooler zones with larger radii, would provide only a very small contribution

TABLE 3

BK Lyn, V751 Cyg, AND V380 OPH LiNe IDENTIFICATIONS FROM STIS SPECTRA a

\begin{tabular}{|c|c|c|c|c|}
\hline Ion & $\lambda_{\text {rest }}$ & $\begin{array}{c}\text { BK } \\
\text { Lyn- }-\lambda_{\text {obs }}\end{array}$ & $\begin{array}{c}\mathrm{V} 751 \\
\text { Cyg- } \lambda_{\text {obs }}\end{array}$ & $\begin{array}{c}\mathrm{V} 380 \\
\text { Oph- } \lambda_{\text {obs }}\end{array}$ \\
\hline C III . & 1175.71 & $1175.39 \mathrm{e}$ & $1175.70 \mathrm{a}$ & $1175.00 \mathrm{e}$ \\
\hline $\mathrm{NV} \quad \ldots \ldots \ldots$ & 1238.82 & $1238.57 \mathrm{e}$ & $1239.29 \mathrm{a}$ & $1237.14 \mathrm{a}$ \\
\hline $1242.80 \ldots \ldots$ & $\ldots$ & $\ldots$ & $1242 \mathrm{a}$ & \\
\hline Si II $\quad \ldots \ldots \ldots$ & 1260.42 & $1260.18 \mathrm{e}$ & $1260.87 \mathrm{a}$ & $1260.35 \mathrm{a}$ \\
\hline O I & 1302.17 & $1302.11 \mathrm{a}$ & $1302.28 \mathrm{a}$ & $1303.51 \mathrm{a}$ \\
\hline $\mathrm{Fe} \mathrm{V} \quad \ldots \ldots \ldots$ & 1329.69 & $1330.52 \mathrm{e}$ & $1330.40 \mathrm{a}$ & \\
\hline C II & 1334.53 & $1334.94 \mathrm{e}$ & & $1334.41 \mathrm{a}$ \\
\hline C II ... & 1335.71 & $\ldots$ & $1334.94 \mathrm{a}$ & \\
\hline $\mathrm{O} v \ldots$ & 1371.29 & $1371.49 \mathrm{e}$ & $1372.25 \mathrm{a}$ & $1335.7077 \mathrm{a}$ \\
\hline $\mathrm{Fe} v \ldots \ldots$ & 1376.34 & $1376.16 \mathrm{e}$ & $1375.75 \mathrm{a}$ & $\ldots$ \\
\hline Si IV $\ldots \ldots$. & 1393.76 & $\ldots$ & $1393.74 \mathrm{a}$ & $1395.48 \mathrm{e}$ \\
\hline Si IV $\quad \ldots \ldots$. & 1402.77 & $\ldots$ & $\ldots$ & $1403.5 \mathrm{e}$ \\
\hline $\mathrm{Fe} \mathrm{V} \ldots \ldots \ldots$ & 1479.17 & $\ldots$ & $1477.17 \mathrm{a}$ & $\ldots$ \\
\hline \multirow[t]{2}{*}{ C IV } & 1548.20 & $1548.26 \mathrm{e}$ & $1544.75 \mathrm{a}$ & $1548 \mathrm{e}$ \\
\hline & 1550 & $\ldots$ & $\ldots$ & $1550.67 \mathrm{e}$ \\
\hline C I & 1560.68 & $\ldots$ & $1560.48 \mathrm{a}$ & $\cdots$ \\
\hline He II & 1640.47 & $1639.86 \mathrm{e}$ & $1645.61 \mathrm{a}$ & $1641.41 \mathrm{e}$ \\
\hline ......... & 1657.01 & . & $1657.16 \mathrm{a}$ & $\ldots$ \\
\hline Si I $\quad \ldots \ldots \ldots$ & 1696.16 & $1696.29 \mathrm{e}$ & $1696.20 \mathrm{a}$ & $\ldots$ \\
\hline
\end{tabular}

${ }^{\mathrm{a}} \mathrm{a}$-denotes an absorption feature, e-denotes an emission feature. to the mid and far-UV disk flux, particularly the SWP FUV bandpass. The mass-transfer rate is assumed to be the same for all radii. Thus, the run of disk temperature with radius is taken to be

$$
T_{\text {eff }}(r)=T_{s} x^{-3 / 4}\left(1-x^{-1 / 2}\right)^{1 / 4},
$$

where $x=r / R_{\mathrm{wd}}$ and $\sigma T_{s}^{4}=3 G M_{\mathrm{wd}} \dot{M} / 8 \pi R_{\mathrm{wd}}^{3}$

The disk is divided into a set of ring annuli. The vertical structure of each ring is computed with TLUSDISK (Hubeny 1990), which is a derivative of the stellar atmosphere program TLUSTY (Hubeny 1988). The spectrum synthesis program SYNSPEC described by Hubeny \& Lanz (1995) is used to solve the radiative transfer equation to compute the local, rest-frame spectrum for each ring of the disk. In addition to detailed profiles of the $\mathrm{H}$ and $\mathrm{He}$ lines, the spectrum synthesis includes metal lines up to $\mathrm{Ni}(\mathrm{Z}=28)$. The accretion disks are computed in LTE and the chemical composition of the accretion disk is kept fixed at solar values in our study.

Limb darkening of the disk is fully taken into account in the manner described by Diaz et al. (1996) involving the Eddington-Barbier relation, the increase of kinetic temperature with depth in the disk, and the wavelength and temperature dependence of the Planck function.

The details of our $\chi_{\nu}^{2}$ (per degree of freedom) minimization fitting procedures are discussed in detail in Sion et al. (1995). We take any reliable published parameters like the WD mass, or orbital inclination only, as an initial guess in searching for the best-fitting accretion disk models. We relax all constraints except the lower limit Knigge (2006) distance. For each system's spectrum, we carry out fits for every combination of $\dot{M}$, inclination, and WD mass in the Wade \& Hubeny (1998) library. The values of $i$ are $18,41,60,75$, and $81^{\circ}$. The range of accretion rates covers $-10.5<\log \dot{M}<-8.0$ in steps of 0.5 in the $\log$ and five different values of the WD mass, namely, $0.4,0.55,0.8$, 1.0 , and $1.2 M_{\odot}$. Out of the roughly 900 models using every combination of $i, \dot{M}$, and $M_{w d}$, we try to isolate the best-fitting accretion disk model. In effect, we search for the best fit based upon (1) the minimum $\chi^{2}$ value achieved, (2) the goodness of fit of the continuum slope, (3) the goodness of fit to the observed Ly $\alpha$ region, and (4) consistency of the scale factor-derived distance with the adopted distance or distance constraint. In other words, the fitting solution may not necessarily be the model with the lowest $\chi^{2}$ value, but rather all other available constraints are used such as constraints on the distance; reliable system parameters if available;and absorption line fits, especially the Ly $\alpha$ wings but sometimes even Si II, Si III, C III, C II, Si IV, and $\mathrm{Si}$ II when these features are in absorption and do not have an origin in a wind or corona. We also search for any statistically significant improvement in the fitting by combining WD models and disk models together using a $\chi^{2}$ minimization routine called DISKFIT. Once again, we find the minimum $\chi^{2}$ value achieved for the combined models, and check the combined model 
consistency with the observed continuum slope and Ly $\alpha$ region, and consistency of the scale factor-derived distance with the adopted distance or distance constraints. For the WD radii, we use the mass-radius relation from the evolutionary model grid of Wood (1990) for C-O cores.

\section{THEORETICAL MODEL FITTING RESULT}

For a nonmagnetic nova-like variable during its high state, it is reasonable to expect that a steady state optically thick accretion disk might provide a successful fit (see Hamilton \& Sion 2008). This was our intial expectation for all three nova-likes, BK Lyn, V380 Oph, and V751 Cygni.

For BK Lyn, the most striking feature of its spectrum is the sharp, narrow Ly $\alpha$ line in combination with a steeply rising continuum. The narrowness of the $\operatorname{Ly} \alpha$ and steep continuum slope suggested the possiblity that the FUV flux originates from a hot WD photosphere. But a fit to the the continuum slope with a hot photosphere at $T=30000 \mathrm{~K}$ and a nominal WD gravity of $\log$ $g=8.0$ yields a synthetic Ly $\alpha$ absorption feature which is far too broad. However, a narrower profile is obtained by increasing the effective temperature and/or lowering the gravity. The continuum slope and narrow $\operatorname{Ly} \alpha$ feature of the observed spectrum can be matched with a WD photosphere with $T=38000 \mathrm{~K}$ and $\log g=7$. A surface gravity this low would not be inconsistent with the low WD mass derived by Dobrzycka \& Howell (1992).
TABLE 4

Accretion Disk Model Fitting to the STIS Spectra of BK LyN

\begin{tabular}{cccccc}
\hline \hline$M_{\text {wd }}\left(M_{\odot}\right)$ & $\dot{M}_{\text {model }}\left(M_{\odot} \mathrm{yr}^{-1}\right)$ & $\begin{array}{c}i \\
\left({ }^{\circ}\right)\end{array}$ & $\chi^{2}$ & $\begin{array}{c}d \\
(\mathrm{pc})\end{array}$ \\
\hline 1.2 & $\ldots \ldots$ & -9.0 & $75-81$ & $1.88-2.05$ & $300-900$ \\
1.0 & $\ldots \ldots$ & -9.0 & $75-81$ & 1.94 & $400-700$ \\
0.8 & $\ldots \ldots$ & -8.5 & $75-81$ & $1.97-1.99$ & $560-940$ \\
0.55 & $\ldots \ldots$ & -8.0 & $75-81$ & $1.97-2.00$ & $730-1220$ \\
0.40 & $\ldots \ldots$ & -8.0 & 75 & 2.06 & 853 \\
\hline
\end{tabular}

However, the distance implied by the fit is $1400 \mathrm{pc}$ while the stellar radius approached that of a hot subdwarf. Moreover, the V-band magnitude of this model fit is 20.6, which is about five magnitudes fainter than the observed magnitude.

The narrowness of the Ly $\alpha$ profile also suggested the possibility that it is of interstellar origin. First, if the system were being viewed at low inclination, then the emission lines, which are presumed to be formed in the disk, would not be as broad as observed. This makes it likely that the narrow Ly $\alpha$ absorption, given that it is not due to a low gravity WD (see previous paragraph), is due to interstellar atomic hydrogen. The reddening toward BK Lyn is rather small, possibly as small as $\sim 0.01$ but the corresponding density is enough to produce such an absorption. One needs an $\mathrm{H}$ column density of only $\sim 1 \times 10^{19} \mathrm{~cm}^{-2}$,

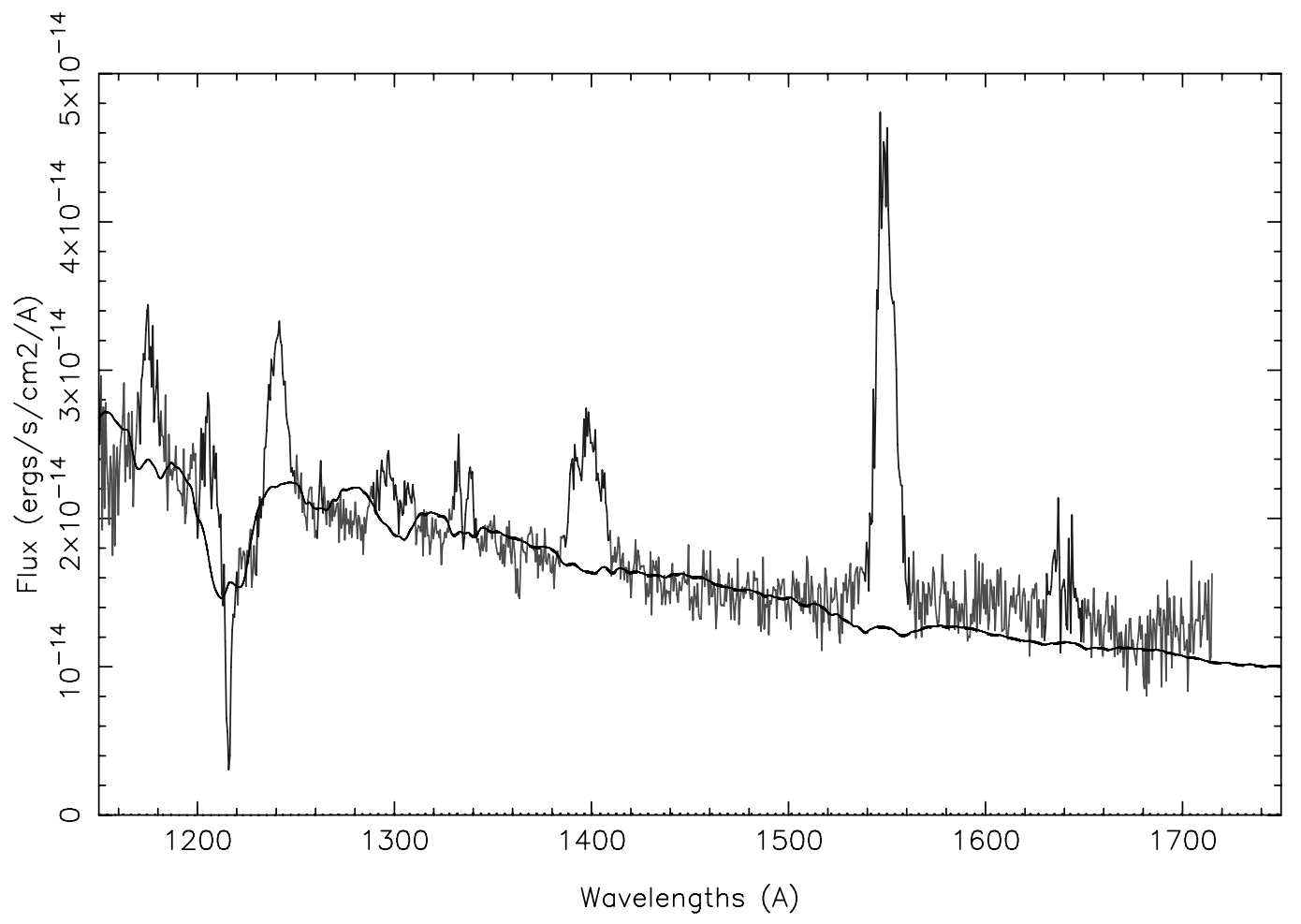

FIG. 2.-Best fitting accretion disk model fit to the Hubble STIS spectrum of BK Lyn. This fit yields $\dot{M}=1 \times 10^{-8.5}$ with $d=560 \mathrm{pc}, \mathrm{M}_{\mathrm{wd}}=0.8 \mathrm{M}_{\odot}$. See the electronic edition of the PASP for a color version of this figure. 


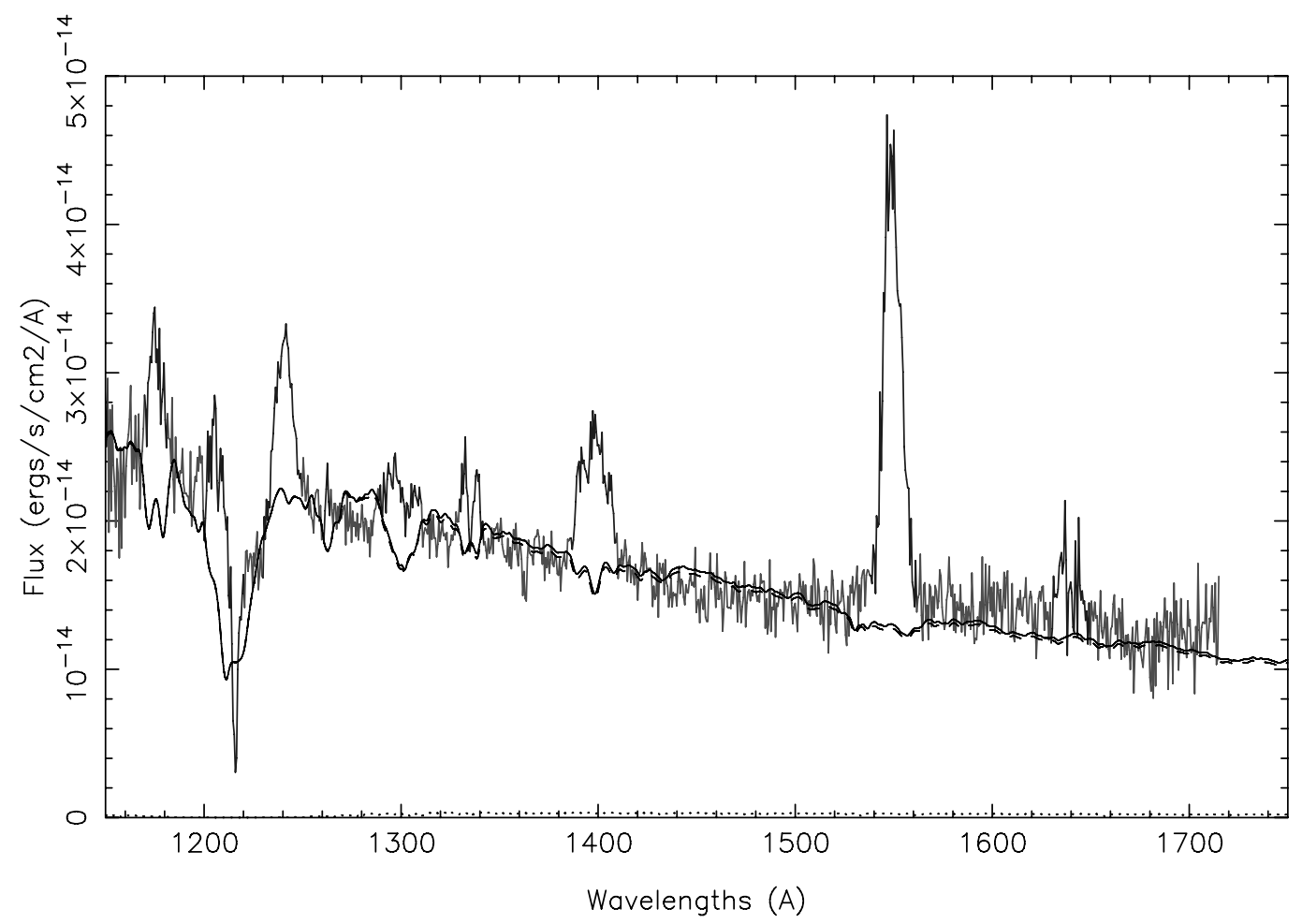

FIG. 3.-Best fitting accretion disk model fit to the Hubble STIS spectrum of BK Lyn. This fit yields the parameters $d=860 \mathrm{pc}, M_{\text {wd }}=0.4 M_{\odot}, i=75^{\circ}$, and $\dot{M}=1 \times 10^{-8}$. See the electronic edition of the PASP for a color version of this figure.

which is a small fraction of the galactic column in line of sight to BK Lyn, which is $\sim 1.2 \times 10^{20} \mathrm{~cm}^{-2}$.

Since a WD photosphere is unlikely to provide the FUV flux, we tried fitting accretion disk models alone to the STIS data. Because the narrow Ly $\alpha$ line is almost certainly interstellar, the mass of the WD, inclination angle and distance are essentially unconstrained except for the lower limit to the distance given by the Knigge (2006) method. Moreover, the presence of broad emission lines and the optical radial velocity amplitude of $\pm 100 \mathrm{~km} \mathrm{~s}^{-1}$ rules out a face-on or very low inclination system. Applying our fitting procedures described in $\S$, we found that accretion disk models yielded much better fits in every respect than a WD photosphere. We also tried WD plus accretion disk combination fits but we found that the best fits resulted only when the WD was contributing $1 \%$ or less of the FUV flux. Thus our best-fitting solutions result when the WD flux contribution is negligible. In Table 4 we have summarized the disk model fitting results to the spectrum of BK Lyn.

In Figures 1, 2 and 3, we display the best-fitting accretion disk models for a $1.2 M_{\odot} \mathrm{WD}$, a $0.8 M_{\odot} \mathrm{WD}$, and a $0.4 M_{\odot}$ WD. We find that the disk fitting favors high inclination with the accretion rate of BK Lyn lying between $\dot{M}=1 \times$ $10^{-8} M_{\odot} \mathrm{yr}^{-1}$ and $\dot{M}=1 \times 10^{-9} M_{\odot} \mathrm{yr}^{-1}$ for the highest $\left(1.2 M_{\odot}\right)$ and lowest $\left(0.4 M_{\odot}\right)$ WD mass, respectively. It is likely that at this inclination of $i=75^{\circ}$, the WD could be masked by the swollen disk. Moreover, an inclination this high cannot be ruled out given how poorly known it appears to be from radial velocity studies.

Applying the same fitting procedures to V751 Cygni, we found no combination of parameters in the accretion disk model grid which provided any acceptable agreement with the dereddened $(\mathrm{E}(B-V)=0.25)$ STIS spectrum of V751 Cygni. The only solution that even remotely resembled the STIS spectrum (but still in very poor agreement) is an accretion disk model with a WD mass, $M_{\mathrm{wd}}=0.55 M_{\odot}$, an orbital inclination $i=75^{\circ}$, a distance of only $78 \mathrm{pc}$, with an accretion rate of $\dot{M}=1 \times$ $10^{-9} M_{\odot} \mathrm{yr}^{-1}$. The $\chi_{\nu}^{2}$ value for this fit is $\chi_{\nu}^{2}=11.94$. Increasing the dereddening value up to 0.5 decreases the distance even more. Thus we are forced to conclude that there is no optically thick steady state disk model that fits the FUV spectrum of V751 Cygni. In Figure 4, we display the STIS spectrum of V751 Cygni together with an accretion disk model that illustrates the extent of the disagreement between standard disk models and the observed data.

Unfortunately, the situation is only slightly better for V380 Oph. We encountered the same difficulty finding acceptable fits. As stated earlier, this system also has the same inconsistency between the observed Ly $\alpha$ profile width and the observed continuuum slope as we found for V751 Cyg. This led us to first explore the effect of interstellar reddening on V380 Oph's spectrum. In our disk model fitting therefore we treated $\mathrm{E}(B-V)$ as a free parameter. We found that for a color excess 


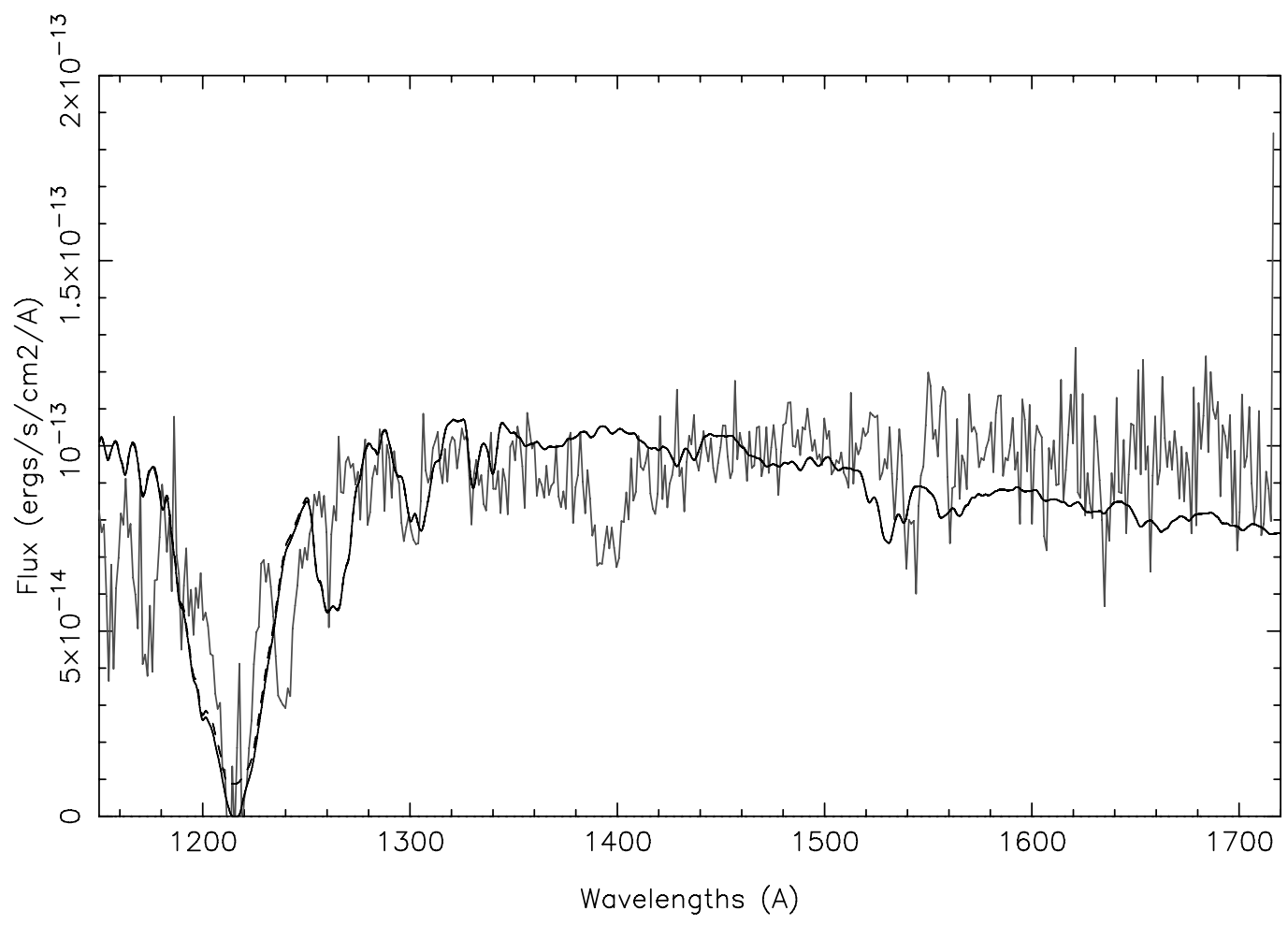

FIG. 4.-Comparison of a standard accretion disk model with the dereddened Hubble STIS spectrum of V751 Cygni during its high optical brightness state illustrating the extent of disagreement. The disk model has $M_{\mathrm{wd}}=0.55 M_{\odot}, i=75^{\circ}, \dot{M}=1 \times 10^{-9} M_{\odot} \mathrm{yr}^{-1}$, but a distance of only 78 pc! See the electronic edition of the $P A S P$ for a color version of this figure.

$\mathrm{E}(B-V)=0.20$, the observed spectral slope and observed Ly $\alpha$ improved slightly but considerable inconsistency remained. As in the case of V751 Cygni, we carried out disk model fits to V380 Oph for every combination of $\dot{M}$, inclination, and WD mass in our disk model library. The only model fit to the STIS data that even slightly resembled (but still in poor agreement with) the STIS spectrum was an accretion disk model alone. For $\mathrm{E}(B-V)=0.20$, this closest but still rather poor fit corresponded to $M_{\mathrm{wd}}=0.4 M_{\odot}, i=75^{\circ}$ with $\dot{M}=1 \times 10^{-8} M_{\odot} \mathrm{yr}^{-1}$ for a $\chi_{\nu}^{2}=13.76$ and a distance of 312 pc. In Figure 5, we illustrate the extent of the disagreement between the STIS spectrum of V380 Oph and a standard accretion disk model. The fitting of the Ly $\alpha$ absorption feature has been achieved with the inclusion of the ISM model. The ISM model has an hydrogen column density of $3 \times 10^{20} \mathrm{~cm}^{-2}$, a turbulent velocity of $50 \mathrm{~km} \mathrm{~s}^{-1}$ and a temperature of $T=170 \mathrm{~K}$. Without the ISM model (dashed line) the disk model does not fit the bottom of the $\operatorname{Ly} \alpha$ profile.

It is clear that for V380 Oph, a definite SW Sex star, and for V751 Cygni (itself is a possible SW Sex member), optically thick steady state accretion disk models simply cannot be fit successfully to their FUV spectra. If one examines their FUV to optical spectral energy distribution, both V380 Oph and V751 Cygni look too red for a standard accretion disk SED in terms of UV-optical color. On the other hand, such a conclusion may not apply to all definite SW Sex stars. The results of Ballouz \& Sion (2009) suggest that for some definite SW Sex stars in their high states, optically thick, steady state disk models do provide a fair representation of their FUV energy distribution and $\operatorname{Ly} \alpha$ regions.

\section{SUMMARY}

We obtained and analyzed the first FUV spectrum ever taken of BK Lyn, the only confirmed nova-like variable known to be below the $\mathrm{CV}$ period gap. The spectrum is characterized by a steeply rising continuum toward shorter wavelengths together with strong broad emission features at C III (1175), NV (1240), Si III + OI (1300), C II (1335), Si IV (1400), C IV (1550), and weak He II (1640) emission. As noted earlier, the emission lines and continuum slope in BK Lyn's spectrum are similar in appearence to the HST and HUT spectra of the dwarf nova SS Cygni and to the FUV spectrum of V794 Aql in its high state. The emission lines are presumed to have formed in an accretion disk present in the system at the time of our HST spectrum. A very narrow Ly $\alpha$ absorption feature is seen which is almost certainly due to interstellar hydrogen. No direct evidence of wind outflow in the form of P Cygni-type profiles is seen in the $H S T$ spectrum. With our steady state 


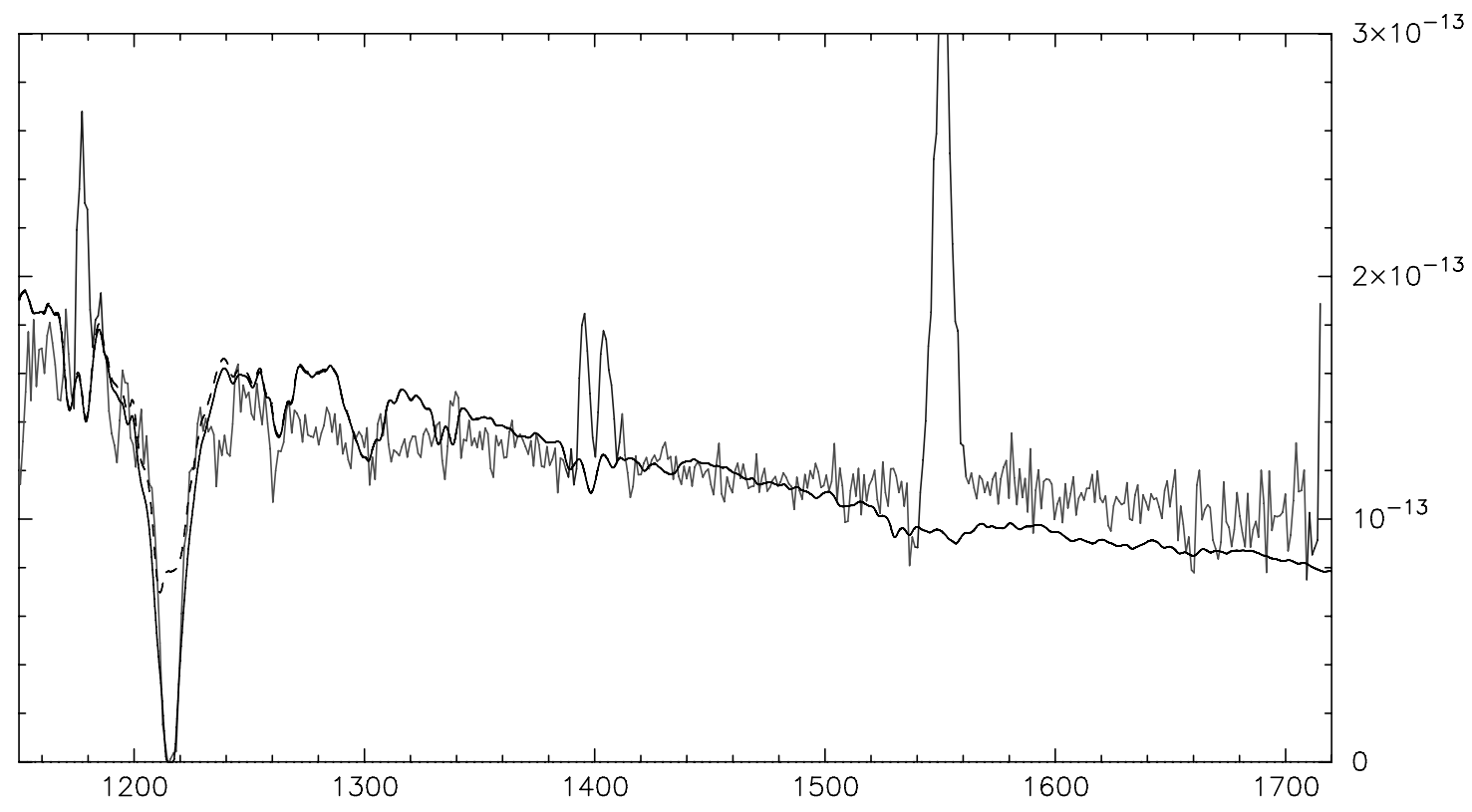

FIG. 5.-Comparison of a standard accretion disk model with the dereddened Hubble STIS spectrum of V380 Oph during its high optical brightness state illustrating the extent of disagreement. The disk model has $M_{\mathrm{wd}}=0.40 M_{\odot}, i=75^{\circ}, \dot{M}=1 \times 10^{-8} M_{\odot} \mathrm{yr}^{-1}$, and a distance of $312 \mathrm{pc}$. The fit to the Ly $\alpha$ profile has been achieved with the inclusion of an ISM model (solid line). The disk model alone, without the ISM inclusion, does not reach the bottom of the Ly $\alpha$ feature (dashed line). See the electronic edition of the PASP for a color version of this figure.

models, we find that BK Lyn has an accretion rate in the range $\dot{M}=1 \times 10^{-9} M_{\odot} \mathrm{yr}^{-1} \quad$ if $\quad M_{\text {wd }}=1.2 M_{\odot} \quad$ and $\dot{M}=1 \times$ $10^{-8} M_{\odot} \mathrm{yr}^{-1}$ if $M_{\mathrm{wd}}=0.4 M_{\odot}$.

If mass transfer in $\mathrm{CV}$ systems below the period gap is driven by angular momentum loss due to gravitational wave emission alone, then the predicted accretion rate at an orbital period of 108 minutes is $5 \times 10^{-11} M_{\odot}$ (Howell et al. 2001), a value much lower than the likely average accretion rate of a nova-like system like BK Lyn.

Puebla et al. (2007) noted the peculiarity of BK Lyn and stated that there was no way to fit the HST/STIS spectrum of BK Lyn with their accretion disk model. They pointed out that it is possible BK Lyn has an optically thin disk with a very low accretion rate. However, they adopted a distance of only $d=150 \mathrm{pc}$ (presumably the average of Sproat's distance of $114 \mathrm{pc}$ and Dhillon's distance of $185 \mathrm{pc}$ ) and they determined an accretion rate of $3.3 \times 10^{-10} M_{\odot} \mathrm{yr}^{-1}$. Taking their low accretion rate, BK Lyn should be a dwarf nova. However, an outburst has never been observed either by ROBOSCOPE, Rotse, or by the AAVSO and Vsnet.

Nova-like variables are generally associated with higher average mass-transfer rates than dwarf novae. Indeed, an examination of the WD temperatures in CVs below the period gap are strongly clustered around 15,000 K (Szkody et al. 2002; Gänsicke et al. 2005) with the hottest example being VW Hyi with $T_{\text {eff }}=20,000 \mathrm{~K}$. Since there is as yet no evidence of lowbrightness states in BK Lyn, the accretion rate may remain high for long intervals of time. Therefore, in view of the higher average mass-transfer rate compared with dwarf novae below the gap, it would be most interesting to know if the underlying WD's surface temperature, which is controlled by long-term compressional heating, is hotter than the nominal $15,000 \mathrm{~K}$ which is typical of WDs in CVs below the gap.

For V751 Cygni, a VY Scl-type nova-like variable, we carried out an analysis of the dereddened HST STIS spectrum and an archival IUE spectrum obtained near maximum brightness, using the lower limit distance obtained by the Knigge (2006) method to help constrain the fits. The fact that the FUV spectrum is dominated by absorption features suggests that the inclination may be low. If, according to Greiner et al. (1999), it is $i=30 \pm 20$ degrees, then it is very likely that one is observing at least some fraction of the upper hemisphere of the WD as well as the dominant luminous accretion disk. Therefore, it would most interesting to gain an estimate of the WD temperature. Unfortunately, during the high state of a nova-like variable viewed at low inclination, the accretion disk light normally dominates and it is difficult to constrain the temperature of the accreting WD without spectra such as those obtained with FUSE, which extend down to the Lyman limit where in some cases a disk and photosphere present distinguishable signatures. The slope of the continuum in V751 Cygni is relatively flat even after the large dereddening while the Ly $\alpha$ absorption is narrow. We were unable to find any optically thick, steady state accretion disk model that would yield a satisfactory fit to the 
STIS spectrum of V751 Cygni. Therefore, we could not determine a reliable accretion rate for this system. As stated earlier, V751 Cygni could turn out to be an SW Sextantis subclass of nova-like variables. Many such systems reveal reddish UV-optical spectral energy distributions and thus cannot be fit with standard disk models.

For V380 Oph, a definite SW Sextantis nova-like system, the FUV spectrum is dominated by broad emission features, a relatively flat continuum, and a narrow $\operatorname{Ly} \alpha$ absorption feature. Even with a reddening of $\mathrm{E}(B-V)=0.2$ and higher, our synthetic spectral analysis using standard accretion disk models was unable to derive a reliable accretion rate for V380 Oph. This is another example of an SW Sex star with a reddish UV-optical spectral energy distribution that cannot be fit successfully with standard disk models. Like V751 Cygni, we can say little about the WD in V380 Oph during its high state without FUV spectra which extend down to the Lyman limit. Further determinations of accretion rates and, whenever possi- ble, WD temperatures are required to elucidate the role of SW Sex stars and other nova-like variables in CV evolution.

This work was supported by HST snapshot grants GO-09357.02A and GO-9724.02A, NSF grant AST0807892, NASA grant NNG04GE78G, and by summer undergraduate research support from the NASA-Delaware Space Grant Consortium. The ISM model used in this work was generated by Paul E. Barrett (USNO) for the analysis of the FUSE spectra of DNs and NLs related to a different project (P.I. Godon). P.G. wishes to thank Mario Livio for his kind hospitality at the Space Telescope Science Institute (STSci), where part of this work was carried out. Some or all of the data presented in this article were obtained from the Multimission Archive at STSci (MAST). STScI is operated by the Association of Universities for Research in Astronomy, Inc., under NASA contract NAS526555. Support for MAST for non- $H S T$ data is provided by the NASA Office of Space Science via grant NAG5-7584 and by other grants and contracts.

\section{REFERENCES}

Araujo-Betancor, S., Gänsicke, B. T., Long, K. S., Beuermann, K., de Martino, D., Sion, E. M., \& Szkody, P. 2005, ApJ, 622, 589

Ballouz, R. L., \& Sion, E. M. 2009, ApJ, in press

Bruch, A., \& Engel, A. 1994, A\&AS, 104, 79

Dhillon, V. S., Littlefair, S. P., Howell, S. B., Ciardi, D. R., Harrop-Allin, M. K., \& Marsh, T. R. 2000, MNRAS, 314, 826

Diaz, M. P., Wade, R., \& Hubeny, I. 1996, ApJ, 459, 236

Dobrzycka, D., \& Howell, S. B. 1992, ApJ, 388, 614

Downes, R., Hoard, D. W., Szkody, P., \& Wachter, S. 1995, AJ, 110,1824

Echevarría, J., Costero, R., Tovmassian, G., Zharikov, S., Pineda, L., \& Michel, R. 2002, Rev. Mex. AA Ser. Conf., ed. W. Henney, J. Franco, M. Martos, \& M. Pena 12, 86

Gänsicke, B. T., Szkody, P., Howell, S. B., \& Sion, E. M. 2005, ApJ, 629,451

Godon, P., Sion, E. M., Barrett, P. E., \& Szkody, P. 2007, ApJ, 656, 1029

Greiner, J. 1998, A\&A, 336, 626

Greiner, J., Tovmassian, G., DiStefano, R., Prestwich, A., Gonzalez-

Riestra, R., Szentasko, L., \& Chavarria, C. 1999, A\&A, 343, 183

Hamilton, R. T., \& Sion, E. M. 2008, PASP, 120, 165

Hoard, D., et al. 2003, AJ, 126, 2473

Howell, S. B., Nelson, L. A., \& Rappaport, S. 2001, ApJ, 550, 897

Hubeny, I. 1988, Comput. Phys. Comm., 52, 103 1990, ApJ, 351, 632

Hubeny, I., \& Lanz, T. 1995, ApJ, 439, 875

Knigge, C. 2006, MNRAS, 373, 484

La Dous, C. 1991, A\&A, 252, 100
Livio, M., \& Pringle, J. E. 1994, ApJ, 427, 956

Long, K. S., Froning, C. S., Knigge, C., et al. 2005, ApJ, 630, 511

Patterson, J., Thorstensen, J. R., Fried, R., Skillman, D., Cook, K., \& Jensen, K. 2001, PASP, 113, 72

Puebla, R. E., Diaz, M. P., \& Hubeny, I. 2007, AJ, 134, 1923

Ringwald, F. A., Thorstensen, J. R., Honeycutt, R. K., \& Robertson, J. W. 1996, MNRAS, 278, 125

Rodriguez-Gil, P., Schmidtobreick, L., \& Gänsicke, B. T. 2007, MNRAS, 374, 1359

Shafter, A. W. 1983, Ph.D. thesis, Univ. California, Los Angeles

Shugarov, S. Yu., Katysheva, N. A., Seregina, T. M., Volkov, I. M., \& Kroll, P. 2005, in The Astrophysics of Cataclysmic Variables and Related Objects, ASP Conf. Proc. 330, ed. J.-M. Hameury, \& J.-P. Lasota (San Francisco: ASP), 495

Sion, E. M., Huang, M., Szkody, P., \& Cheng, F. 1995 ApJ, 444, 97

Skillman, D. R., \& Patterson, J. 1993, ApJ, 417, 298

Sproats, L. N., Howell, S. B., \& Mason, K. O. 1996, MNRAS, 282, 1211

Szkody, P., Sion, E. M., Gänsicke, B. T., Howell, S. B. 2002, ASPC, 261, 21

Verbunt, F. 1987, A\&AS, 71, 339

Wade, R. A., \& Hubeny, I. 1998, ApJ, 509, 350

Walker, M., \& Bell, M. 1980, BAAS, 12, 63

Warner, B. 1995, Cataclysmic Variable Stars (Cambridge: Cambridge Univ. Press)

Wood, M. A. 1990, Ph.D. thesis, University of Texas at Austin Wu, K., Wickramasinghe, D., \& Warner, B. 1995, PASA, 12, 604 